\title{
ACNE CONGLOBATA ASSOCIATED WITH HIDRADENITIS SUPPURATIVA, DISORDERS OF FOLLICULAR OCCLUSION (CASE REPORT)
}

\author{
Pecova K, jr. \\ Department of of Dermatovenerology, Comenius University, Jessenius Faculty of Medicine in Martin and \\ University Hospital in Martin, Slovakia
}

\begin{abstract}
The author is presenting the case of a 23-year-old female patient with a severe form of acne conglobata, with the first symptoms of the disease occurring as far back as the prepubertal age. In the past year the disease has combined with hidradenitis suppurativa (to be referred to henceforth as "HS"), Hurley stage I, in the axillae and both sides of the inguinal region, with a family history of acne conglobata (both her mother and brother were affected). Further examinations ruled out inflammatory bowel disease because of a lack of further associated symptoms, except for sideropenic anaemia (lesser form) and lower serum values of vitamin D. Up until now the disease has been resistant to treatment, including the long-term treatment of methylprednisolone in combination with isotretinoid as well as dapsone and antibiotics.
\end{abstract}

Key words: acne conglobata, hidradenitis suppurativa, treatment

\section{INTRODUCTION}

Hidradenitis suppurativa (HS) may occur in combination with a severe form of acne (acne conglobata), dissecting cellulitis of the scalp and pilonidal sinus (pilonidal cysts) [1].

We present a case of the simultaneous occurrence of the symptoms of severe acne conglobata and HS.

\section{Case report}

A 23-year old female patient (175 cm tall, $60 \mathrm{~kg}$ weight, BMI -19.2), mother of two children, currently on maternal leave, with smoking being ruled out, and with her mother and brother having been treated for severe symptoms of acne conglobata.

The patient is being monitored and treated for sideropenic anaemia. The patient feels weak, tired, and reports general joint pain as well as intensive pain from individual lesions of acne conglobata radiating outwards. Her medical history includes occasional gastric pain and occasional cramps in the hypogastric region, associated with diarrhoea without the presence of mucus and blood and intermittent subfebrile rises in temperature. From the age of 12 , at the time of her first period, the patient reports deep subcutaneous nodes, with subsequent purulent production in the face, back of the head, back and in the decollete area, classified by dermatologists as acne conglobata. Last year similar lesions classified as abscesses and fistulas producing pus also appeared in the inguinal area and less numerous in the hypogastric area and in the axillary areas on both sides of the body. Upon examination, the clinical symptoms were re-classified as HS, Hurley stage I (Fig. 1, 2) with symptoms of acne conglobata (Fig. 3,4). Further investigations were performed, determining that her gynaecological status is adequate for her age with a regular period. The patient has a history of adnexitis, and she currently has an intrauterine device (IUD) in place. ENT examination as well as an X-Ray of the paranasal sinuses ruled out focal infection at these locations; however, dental examination confirmed multiple root granulomas as well as den-

Address for correspondence:

MUDr. Klaudia Pecova, Dept of Dermatovenerology, Jessenius Fcaulty of Medicine in Martin, Comenius University in Bratislava, University Hospital in Martin (03601), Kollarova Str. 2, Slovakia; e-mail: klaudiapec@gmail.com 


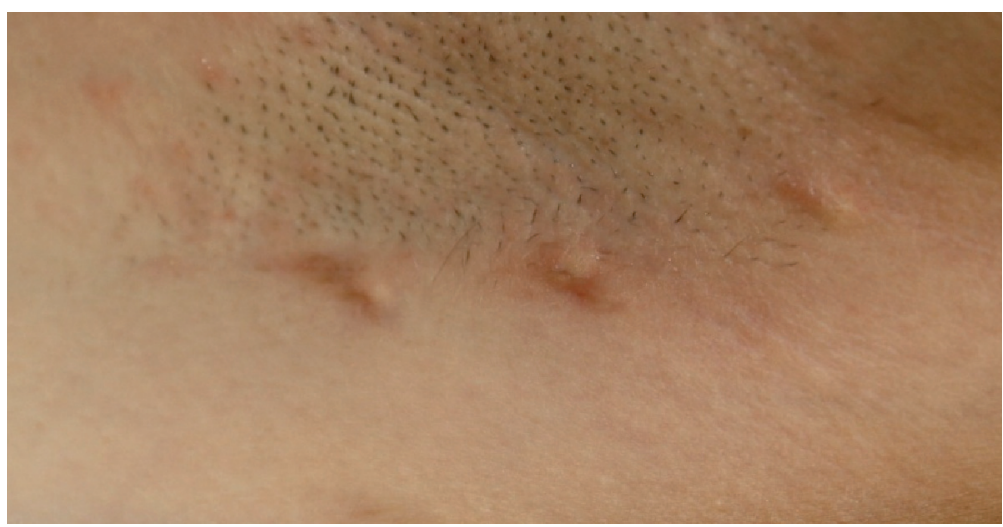

Fig. 1 Symptoms of HS in axillary areas (Hurley I)

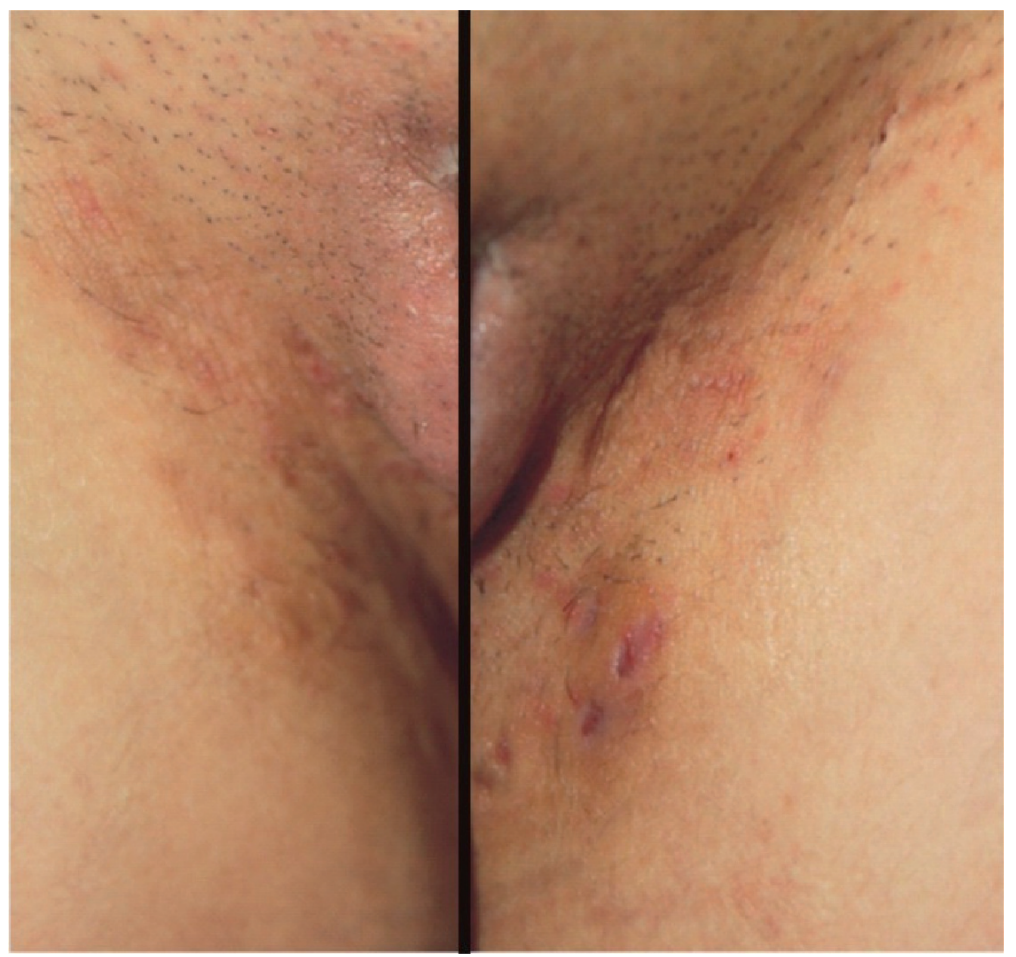

Fig. 2 Symptoms of HS in both inguinal areas, with the upper part of the figure showing a double comedo, a typical symptom of HS.

tal caries. An ultrasound of the abdominal organs (liver, gall bladder, spleen, pancreas, kidneys, paraaortal lymph nodes) and peripheral lymph nodes, as well as an X-Ray of the lungs, failed to demonstrate any pathology. A colonoscopy ruled out an inflammatory disorder of the intestines, but upon examination of the stool for parasites, Ascaris lumbricoides was found and subsequently treated with mebendazole. A gastrofibroscopy confirmed the lack of a Helicobacter pylori infection. The patient had thrombophlebitis of the right median cubital vein along a $6 \mathrm{~cm}$ segment, treated with fraxiparine. Cultures from acne conglobata lesions on the patient's back repeatedly yielded Methicilin/oxacilin resistant 


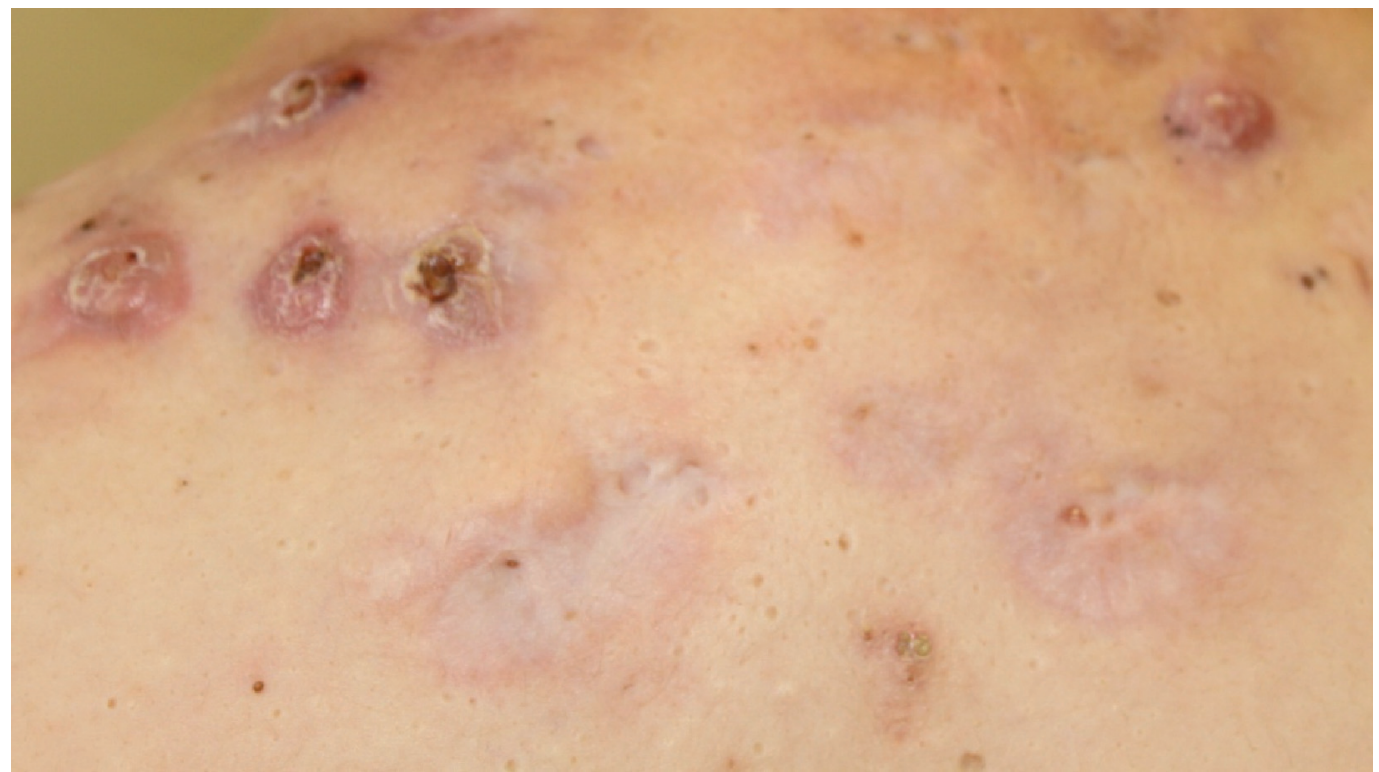

Fig.3 The area surrounding the lesions is inflamed, with several simple comedones, typical of acne.

Staphylococcus aureus (MRSA), and on other occasions only MRSA negative Staphylococcus aureus, Pseudomonas stutzeri, Streptococcus agalactiae and anaerobic Propionbacterium acnes. The patient's urine tested positive for MRSA Staphylococcus aureus, and subsequently for Staphylococcus aureus - MRSA negative, Streptococcus agalactiae and Escherichia coli. From the patient's vagina, MRSA was also first detected, and Staphylococcus aureus, Lactobacillus species and Candida albicans were later detected in the patient's stool.

In the patient $\mathrm{s}$ biochemical profile the following results were within reference range: minerals (Na, K, Cl, P, Ca, Mg), ferritin, vitamin B12, urea, creatinine, glycaemia, C-peptide, glycated haemoglobin (HbAlc), uric acid, total proteins, homocystein, protein electrophoresis ELFO beta 1, immunoglobulins IgA, IgM, IgG, IgE, light kappa chains and lambda chains and kappa/lambda ratio, absence of paraprotein, C3 and C4 complement components, lipids (total cholesterol, HDL cholesterol, triglycerides), liver enzymes (total bilirubin, GMT, AST, ALT, lactatedehydrogenase, amylase), antistreptolysinO (ASLO), rheumatoid factor, blood cells count, differential cells count as well as erythrocyte sedimentation rate, coagulation parameters - APTT - activated partial thromboplastin time as well as APTT - ratio, methemoglobin as well as normal levels of glucose 6-phosphate dehydrogenase, stool for occult bleeding, pANCA (myeloperoxidase), cANCA (PRO3), ANA antinuclear antibodies, anti-ds DNA, anti-sm, anti-SS-A, anti-SS-B, anti-Scl-70, thyroid hormones (FT3, FT4, TSH, anti-TG, anti-TPO), and negative Quantiferon tests,Yersinia serology,HIV p24Ag, HBsAg, $\mathrm{HCV}$, TPHA as well as cytomegalovirus tests, Epstein-Barr virus tests and borreliosis tests.

Biochemical parameters repeatedly revealed a lower level of serum ironwithin 5.1- 6.7 umol/1 (reference range 7.2 - $21.5 \mathrm{umol} / \mathrm{l}$ ), reduced serum levels of total vitamin D (25$\mathrm{OH}) 15.6 \mathrm{ng} / \mathrm{ml}$ (reference range 30.0 - $50.0 \mathrm{ng} / \mathrm{ml}$ ) and a persisting elevation of creatinephosphokinase (CK) within the range of 2.65 - 3.51 ukat/1 (reference range $0.0-2.42$ ukat/l).

The patient was treated with methylprednisolone at a dose of $0.5 \mathrm{mg} / \mathrm{kg}$ body weight per day in combination with isotretinoid $(0.75 \mathrm{mg} / \mathrm{kg}$ of body weight per day) and metronidazole without any significant effect. At present the patient is being treated with dapsone at 
$100 \mathrm{mg}$ per day as well as antibiotics according to the susceptibility of the cultured strains: ciprofloxacin 1.0 g per day, clindamycin $900 \mathrm{mg}$ per day in combination with metronidazole and probiotics, $600 \mathrm{mg}$ clindamycin in combination with $600 \mathrm{mg}$ rifampicin, amoxicillin + clavulanate 2.0 g per day, trimetoprim + sulfonamide 2 tbl $480 \mathrm{mg} 2 \mathrm{x}$ per day, fluconazole $50 \mathrm{mg}$ per day for 7 days and subsequently $150 \mathrm{mg} 1 \mathrm{x}$ per week, and long-term tetracycline antibiotics. In addition to antibiotic therapy, mebendazole was used to treat the Ascaris lumbricoides infection and fraxiparine to treat thrombophlebitis. At present, despite the above treatment, there is repeated reactivation of the symptoms of HS as well as acne conglobata. Therefore we are considering the initiation of treatment with adalimumab and subsequent surgical management of the condition when the symptoms are reduced.

\section{DISCUSSION}

As previously mentioned, in addition to severe symptoms of acne conglobata, dissecting cellulitis (folliculitis) in the scalp and pilonidal sinus (pilonidal cysts), HS is often associated with SAPHO syndrome (sinovitis, acne, pustulosis, hyperostosis and osteitis) [2] in 8\% of those with ulcerative colitis and up to $17 \%$ of those with Crohn s disease [3]. Crohn s disease as well as HS may have a similar clinical symptomatology at the early stages-in the form of various types of perianal or perirectal sinuses [4]. Occasionally, cases of simultaneous HS and pyoderma gangrenosum are described. Because of the frequent simultaneous appearance of these conditions, a discussion will follow regarding the similar or related pathogenesis of these disorders, with several opinions being put forward.

A range of factors plays an important role in the pathogenesis of HS. These include the gradual development of lesions until the development of a full clinical image of HS (primary and secondary changes of the hair follicle), genetic features of the conditions as well as mechanical, hormonal, immunological and other factors. At present, HS is thought to occur as a result of follicular occlusion, classifying HS in the group of follicular occlusion disorders together with acne conglobata, dissecting cellulitis of the scalp and pilonidal cysts sinuses [5].

It seems that HS lesions appear at locations where sebaceous glands may conglomerate with follicular ducts of hair follicles [6]. Plugs result from excessive hormonal stimulation of ductal keratinocytes, resulting in the rupture of the ductal wall as a result of centrifugal pressure in these areas, leading to the release of intraductal follicular content into the deep dermis with subsequent stimulation of the innate immune system. The healing process tries to restore the normal anatomy of the pilosebaceous unit. If the reparation process fails, follicular fragments stimulate three separate reactions [1]. The first reaction involves the inflammatory response to a foreign body, launching an innate immune system response and resulting in purulence, tissue destruction and excessive scarring. The second reaction may involve the production of epithelialized sinuses from the stem cells of the pilosebaceous unit, which survive and destruct [7]. The third reaction is the production of an invasive proliferative gelatinous matter produced mostly by HS, consisting of a gel with inflammatory cells considered to be precursors of epithelialization elements. Continued hormonal stimulation maintains and supports the production of the invasive material and maintains the inflammatory process in the dermis and in the subcutaneous tissue until this material is eliminated. These processes in the dermis and in the subcutaneous tissues result in the clinical signs of follicular occlusion tetrad-ranging from mild to severe lesions.

It is not assumed that the bacterial infection plays a primary pathogenetic role in the development of follicular occlusion tetrad, i.e. HS and acne conglobata. However, the secondary infection of the lesions may play a role in the chronic nature of the process and relapses of the disease and also participate in tissue destruction process.

There appears to be a strong correlation between sexual hormones and follicular occlusion disorders, especially in women. Serum androgen elevation and sensitivity of the target 
cells appear to play a very important role in this process. The role of the FoxO1 androgen receptor is considered to be the most important. Insulin and insulin-like growth factor (IGF1) have an effect on the increasing availability of androgen receptors, with the effects of both being increased by dietary factors [8]. In women, HS mostly starts at the time of the first period and inflammation is re-activated before menstruation, under the affect of androgen progestins such as medroxyprogesterone acetate (MPA) or levonogrestrel. The clinical picture is generally improved during pregnancy and after menopause.

It is assumed that in up to $35-40 \%$ of follicular occlusion disorder cases, including HS and acne conglobata, family history plays an important role in autosomal dominant heredity with variable penetration. [9]. Current research focuses on the molecular genetic aspects of HS in an effort to determine the so-called candidate genes. Different authors report mutations of genes that regulate transmembrane proteins, including gamma-secretase. However, their research varies, and therefore it is assumed that HS is a heterogenous disorder linked perhaps to the mutation of several genes [9].

There are several questions marks remaining in this case of a patient with HS (Hurley stage I) in combination with a severe form of acne conglobata, a normal body mass index (BMI - 19.2), positive family predisposition to acne conglobata, disease onset at the time of the first period, the chronic nature of the patient's symptoms, as well as relapses and the non-responsive nature of the disorder to treatment. Nevertheless the presented case is extremely interesting in the context of the current research, at least from the viewpoint of the genetic examination and further treatment of the patient.

\section{REFERENCES}

1. Margesson, L.J., Danby, F.W.: Hidradenitis suppurativa. Best Pract Research Obstet \& Gynecol 2014; $28: 1013$ $-1027$.

2. Kahn, M.F., Chamot, A.M.: SAPHO syndrome. Rheum Dis Clin North Am 1992; 18(1): 225 - 246.

3. Van der Zee, H.H., van der Woude, C.J., Florencia, E.F. et al.: Hidradenitis suppurativa and inflammatory bowel disease: are they associated? Results of a pilot study. Br J Dermatol 2010; 162(1): 195 - 197.

4. Alikhan, A., Lynch, P.J., Eisen, D.B.: Hidradenitis suppurativa : a comprehensive review. J Am Acad Dermatol 2009; 60(4): 539 - 561.

5. Han, G.: Biologics in dermatology beyond psoriasis. Cutis 2014; 93(5):E21 - E27.

6. Danby, F.W., Jemec, G.B., Marsch, W. et al.: Preliminary findings suggest hidradenitis suppurativa may be due to defective follicular support. Br J Dermatol 2013;168(5): 1034 - 1039.

7. Gniadecki, R., Jemec, G.B.: Lipid raft-enriched stem cell-like keratinocytes in the epidermis, hair follicles and sinus tracts in hidradenitis suppurativa. Exp Dermatol 2004;13(6): 361 - 363.

8. Melnik, B.C., Zouboulis, C.C.: Potentional role of FoxO1 and mTORC1 in the pathogenesis of western dietinduced acne. Exp Dermatol 2013;22(5): 311 - 315.

9. von der Werth, J.M., Williams, H.C., Raeburn, J.A.: The clinical genetics of hidradenitis suppurativa revisited. Br J Dermatol 2000;142: 947 - 953

Received: April, 30, 2015

Accepted: May, 1, 2015 